\title{
THE DE BRANGES-ROVNYAK MODEL
}

\author{
JAMES GUYKER
}

(Communicated by Paul S. Muhly)

\begin{abstract}
A characterization, extending results of A. Beurling, L. de Branges and J. Rovnyak, of those Hilbert spaces of formal power series, which are isometrically equal to a de Branges-Rovnyak (scalar-valued) function space $\mathscr{H}(b)$, is obtained.
\end{abstract}

Let $\mathscr{C}(z)$ denote the Hilbert space of square-summable power series $f(z)=$ $\sum a_{n} z^{n}$ with complex coefficients such that $\|f(z)\|^{2}=\sum\left|a_{n}\right|^{2}$. For a power series $b(z)$ which converges to a function which is bounded by one in the unit disk, the space $\mathscr{H}(b)$ of L. de Branges and J. Rovnyak [4] is the Hilbert space of series $f(z)$ in $\mathscr{C}(z)$ with the property that

$$
\|f(z)\|_{b}^{2}=\sup \left\{\|f(z)+b(z) g(z)\|^{2}-\|g(z)\|^{2}\right\}
$$

is finite, where the supremum is taken over all elements $g(z)$ of $\mathscr{C}(z)$. If $f(z)$ is in $\mathscr{H}(b)$, then so is the series $[f(z)-f(0)] / z$ and, moreover,

$$
\|[f(z)-f(0)] / z\|_{b}^{2} \leq\|f(z)\|_{b}^{2}-|f(0)|^{2} .
$$

The difference-quotient transformation on $\mathscr{H}(b)$ is the contraction $R(0)$ which maps $f(z)$ into $[f(z)-f(0)] / z$. There have been several recent developments $[1,6,7,8]$ with the space $\mathscr{H}(b)$ especially as a canonical model space for contractions on Hilbert spaces.

Let $\mathscr{H}$ be a Hilbert space of formal power series such that the differencequotient inequality

$$
\|[f(z)-f(0)] / z\|_{\mathscr{H}}^{2} \leq\|f(z)\|_{\mathscr{H}}^{2}-|f(0)|^{2}
$$

holds for every element $f(z)$, and let $T$ denote the contraction on $\mathscr{H}$ which sends $f(z)$ into $[f(z)-f(0)] / z$. By the well-known invariant subspace theorem of A. Beurling [2], if $\mathscr{H}$ is contained isometrically in $\mathscr{C}(z)$, then $\mathscr{H}$ is a space $\mathscr{H}(b)$ for some inner function $b$. (Conversely, if $b$ is inner, then $\mathscr{H}(b)$ is contained isometrically in $\mathscr{C}(z))$. More generally, de Branges and Rovnyak showed in [4, Theorem 15] that if equality holds in (2), for every $f$, and the rank of $1-T T^{*}$ is one, then $\mathscr{H}=\mathscr{H}(b)$ isometrically, where $b$ is an extreme

Received by the editors July $18,1989$.

1980 Mathematics Subject Classification (1985 Revision). Primary 47A45, 46E20, 47B38. 
point of the unit ball in the space of bounded analytic functions of the unit disk. (Conversely, if $b$ is an extreme point, then equality holds in (1) for every $f$, and the ranges of $1-R(0) R(0)^{*}$ and $1-R(0)^{*} R(0)$ are spanned by $[b(z)-b(0)] / z$ and $1-b(z) \overline{b(0)}$ respectively). In this note, we obtain a similar result for spaces $\mathscr{H}(b)$ where $b$ is not an extreme point, which answers a question raised in [4, p. 39].

Suppose that $b$ is nonconstant and not an extreme point. Then the range of $1-R(0) R(0)^{*}$ is spanned by $[b(z)-b(0)] / z$, and the range of $1-R(0)^{*} R(0)$ is spanned by 1 and $b$. Moreover, it follows directly from [8, Lemmas 1-3] that if $f(z)=[1-b(z) \overline{b(0)}]\|b(z)\|_{b}^{2}-b(z) \overline{b(0)}$, then $f(0) \neq 0$ and equality holds in (1) for $f$. These properties are sufficient to characterize $\mathscr{H}(b)$.

Theorem. Let $\mathscr{H}$ be a Hilbert space of formal power series which satisfies (2), and suppose that equality holds in (2) for some element with nonzero constant coefficient. If the ranks of $1-T T^{*}$ and $1-T^{*} T$ are 1 and 2 respectively, where $T$ is the difference-quotient transformation on $\mathscr{H}$, then $\mathscr{H}$ is isometrically equal to a space $\mathscr{H}(b)$ where $b$ is not an extreme point.

The following example shows that the above hypothesis on equality in (2) may not be omitted.

Example. Let $\phi$ be a nonconstant bounded analytic function, and, for $r \geq 1$, let $\mathscr{H}=\mathscr{C}(z)$ with the inner product given by

$$
\langle f, g\rangle_{\mathscr{H}}=\left\langle\left(r+T_{\phi} T_{\phi}^{*}\right) f, g\right\rangle,
$$

where $T_{\phi}$ denotes multiplication by $\phi$ on $\mathscr{C}(z)$. It is easily checked that $\mathscr{H}$ is a Hilbert space and, for every $f$, we have that

$$
\begin{aligned}
\|[f(z)-f(0)] / z\|_{\mathscr{H}}^{2} & =\|f\|_{\mathscr{H}}^{2}-|f(0)|^{2}-(r-1)|f(0)|^{2}-\left|\left(T_{\phi}^{*} f\right)(0)\right|^{2} \\
& \leq\|f\|_{\mathscr{H}}^{2}-|f(0)|^{2} .
\end{aligned}
$$

Furthermore, the range of $1-T T^{*}$ is spanned by $[\phi(z)-\phi(0)] / z$, and the range of $1-T^{*} T$ is spanned by 1 and $\phi$. However, equality holds in (2) for $f$ if and only if $(r-1)\langle f, 1\rangle=0=\langle f, \phi\rangle$. Hence, by the theorem, $\mathscr{H}=\mathscr{H}(b)$ isometrically if and only if $r=1$.

The key to the verification of the theorem is the theory of complementation [3] of de Branges: A Hilbert space $\mathscr{P}$ is contained contractively in a Hilbert space $\mathscr{K}$ if $\mathscr{P}$ is a vector subspace of $\mathscr{K}$ and if the inclusion map $i_{\mathscr{S}}$ of $\mathscr{P}$ into $\mathscr{K}$ is a contraction. If $\mathscr{P}$ is contained contractively in $\mathscr{K}$, then the space complementary to $\mathscr{P}$ in $\mathscr{K}$ is the Hilbert space $\mathscr{Q}$ of elements $g$ of $\mathscr{K}$ with the property that

$$
\|g\|_{\mathscr{C}}^{2}=\sup \left\{\|g+f\|_{. \not H}^{2}-\|f\|_{\mathscr{P}}^{2}\right\}
$$

is finite, where the supremum is taken over all $f$ in $\mathscr{P}$. The space $\mathscr{Q}$ is contained contractively in $\mathscr{K}$ and is the unique Hilbert space such that the 
inequality $\|h\|_{\mathscr{H}}^{2} \leq\|f\|_{\mathscr{P} \mathcal{D}}^{2}+\|g\|_{\mathscr{Q}}^{2}$ holds whenever $h=f+g$ is a decomposition of $h$ in $\mathscr{K}$ into $f$ in $\mathscr{P}$ and $g$ in $\mathscr{Q}$, and such that every $h$ in $\mathscr{K}$ admits the unique decomposition $h=\left(i_{\mathscr{P}}^{*} h\right)+\left(i_{\mathscr{Q}}^{*} h\right)$, where $\|h\|_{\mathscr{K}}^{2}=\left\|i_{\mathscr{P}}^{*} h\right\|_{\mathscr{P}}^{2}+\left\|i_{\mathscr{Q}}^{*} h\right\|_{\mathscr{Q}}^{2}$.

By (2), $\mathscr{H}$ is contained contractively in $\mathscr{C}(z)$. Moreover, if $\mathscr{M}$ is the complementary space to $\mathscr{H}$ in $\mathscr{C}(z)$, then multiplication by $z$ is defined and bounded by one on $\mathscr{M}$. The essential result for us will be the following corollary of de Branges's extension [3, Theorem 15] of Beurling's theorem: if multiplication by $z$ is an isometry on $\mathscr{M}$, then $\mathscr{H}$ is isometrically equal to a space $\mathscr{H}(b)$.

To begin the proof of the theorem, let us first observe that $T$ is onto: If the kernel of $T^{*}$ is nontrivial, then it must coincide with the range of $1-T T^{*}$ since the rank of $1-T T^{*}$ is 1 . However, in this case, $T^{*}$ and hence $T$ are partial isometries so that $\operatorname{ker} T=\operatorname{range}\left(1-T^{*} T\right)$ is two-dimensional, which is impossible. Thus the range of $T$ is dense in $\mathscr{H}$. But $T$ has closed range since $T^{*} T$ has closed range ( $T^{*} T$ is the orthogonal direct sum of the identity operator on the kernel of $1-T^{*} T$ and an operator on the finite-dimensional range of $\left.1-T^{*} T\right)$. Therefore, $\mathscr{H}=T \mathscr{H}$.

Note that constants belong to $\mathscr{H}$ since $T\left(1-T^{*} T\right)=\left(1-T T^{*}\right) T$ and $\operatorname{rank}\left(1-T T^{*}\right)<\operatorname{rank}\left(1-T^{*} T\right)$. Hence, since $T$ is onto, $\mathscr{H}$ has the property that $z f(z)$ is in $\mathscr{H}$ whenever $f(z)$ is in $\mathscr{H}$.

Consider the set $\mathscr{D}$ of those elements of $\mathscr{H}$ for which equality holds in (2). By (2) and the parallelogram law, $\mathscr{D}$ is a vector subspace of $\mathscr{H}$; since $T$ is continuous and $\mathscr{H}$ is contained contractively in $\mathscr{C}(z), \mathscr{D}$ is closed. Also by (2), $\mathscr{D}$ contains the kernel of $1-T^{*} T$; by assumption, $\mathscr{D}$ contains an element with nonvanishing constant coefficient which we may assume belongs to the range of $1-T^{*} T$.

Let us suppose first that 1 does not belong to $\mathscr{D}$. Since $\mathscr{H}$ is thus the orthogonal direct sum of the kernel of $1-T^{*} T$ and the span of 1 and some element of $\mathscr{D}$, we have that $\mathscr{H}=T \mathscr{H}=T \mathscr{D}$. Therefore, for $g$ in the space $\mathscr{M}$, complementary to $\mathscr{H}$ in $\mathscr{C}(z)$,

$$
\begin{aligned}
\|g\|_{\mathscr{M}}^{2} & =\sup \left\{\|g+T f\|^{2}-\|T f\|_{\mathscr{P}}^{2}: f \in \mathscr{D}\right\} \\
& =\sup \left\{\left(\|z g+f\|^{2}-|f(0)|^{2}\right)-\left(\|f\|_{\mathscr{P}}^{2}-|f(0)|^{2}\right): f \in \mathscr{D}\right\} \\
& =\sup \left\{\|z g+f\|^{2}-\|f\|_{\mathscr{H}}^{2}: f \in \mathscr{D}\right\} \\
& \leq\|z g\|_{\mathscr{M}}^{2} .
\end{aligned}
$$

As above, since the reverse inequality holds, multiplication by $z$ is an isometry on $\mathscr{M}$, and hence the theorem follows in this case.

Next, suppose that 1 belongs to $\mathscr{D}$. There exists an integer $n \geq 1$ such that $\mathscr{D}$ contains $z^{m}(m=0, \ldots, n-1)$ but not $z^{n}$. (Otherwise $i_{\not H}^{*} z^{m}=z^{m}$ for every $m \geq 0$, and consequently $\mathscr{H}=\mathscr{C}(z)$ isometrically. But then $T^{*}$ would be multiplication by $z$ on $\mathscr{C}(z)$ so that $1-T T^{*}=0$, contradicting its rank hypothesis.) It follows that $T^{* n} 1 \neq z^{n}$ and $\left(1-T T^{*}\right) z^{n-1} \neq 0$, 
but if $n>1$ then $T^{* m} 1=z^{m}$ and $\left(1-T T^{*}\right) z^{m-1}=0$, for every $m=$ $1, \ldots, n-1 \quad\left(\left(1-T^{*} T\right) z^{n}\right.$ is nonzero and orthogonal to the kernel of $T$ since $\left\langle\left(1-T^{*} T\right) z^{n}, 1\right\rangle_{\mathscr{H}}=\left\langle z^{n}, 1\right\rangle_{\mathscr{H}}=\left\langle z^{n}, i_{\mathscr{H}}^{*} 1\right\rangle_{\mathscr{H}}=\left\langle i_{\mathscr{H}} z^{n}, 1\right\rangle=\left\langle z^{n}, 1\right\rangle=0$. Therefore $\left(1-T T^{*}\right) z^{n-1}=T\left(1-T^{*} T\right) z^{n} \neq 0$.).

The $\mathscr{M}$-norm may be simplified in this case. Specifically, we will show that if $f$ is in $\mathscr{H}$ then there exists an element $\hat{f}$ in the kernel of $1-T^{n} T^{* n}$ such that, for every $g$ in $\mathscr{M}$,

$$
\|g+f\|^{2}-\|f\|_{\mathscr{H}}^{2}=\|g+\hat{f}\|^{2}-\|\hat{f}\|_{\mathscr{P}}^{2} \text {. }
$$

Let $f$ be in $\mathscr{H}$, and define constants $c_{0}, \ldots, c_{n-1}$ recursively as follows:

$$
c_{n-1}=-\left\langle f,\left(1-T T^{*}\right) z^{n-1}\right\rangle_{\mathscr{H}} /\left\langle z^{n-1},\left(1-T T^{*}\right) z^{n-1}\right\rangle_{\mathscr{H}} ;
$$

if $n>1$, use

$$
\begin{aligned}
c_{n-k}= & -\left\langle f+\sum_{n-k+1}^{n-1} c_{m} z^{m}, T^{k-1}\left(1-T T^{*}\right) T^{*^{k-1}} z^{n-k}\right\rangle_{\mathscr{H}} \\
& \div\left\langle z^{n-k}, T^{k-1}\left(1-T T^{*}\right) T^{*^{k-1}} z^{n-k}\right\rangle_{\mathscr{H}},
\end{aligned}
$$

for $k=2, \ldots, n$. By the previous paragraph, $T^{*^{k-1}} z^{n-k}=z^{n-1}$, and furthermore $\hat{f}=f+\sum_{0}^{n-1} c_{m} z^{m}$ is orthogonal to the range of $T^{k-1}\left(1-T T^{*}\right) T^{*^{k-1}}$, which is spanned by $T^{k-1}\left(1-T T^{*}\right) T^{*^{k-1}} z^{n-k}$, for every $k=1, \ldots, n$. Since $1-T^{n} T^{* n}=\sum_{1}^{n} T^{k-1}\left(1-T T^{*}\right) T^{*^{k-1}}$, we have that $\hat{f}$ is orthogonal to the range of $1-T^{n} T^{* n}$.

Let $g$ be in $\mathscr{M}$. Then $\left\langle g, z^{m}\right\rangle=\left\langle i_{\mathscr{M}} g, z^{m}\right\rangle=\left\langle g, i_{\mathscr{M}}^{*} z^{m}\right\rangle_{\mathscr{M}}=\langle g, 0\rangle_{\mathscr{M}}=0$ for every $m=0, \ldots, n-1$, so the first $n$ coefficients of $g$ vanish. Let us write $f=\left(\sum_{0}^{n-1} a_{m} z^{m}\right)+z^{n} T^{n} f$ for some constants $a_{m}$ and observe that $\|f\|_{\mathscr{H}}^{2}=\sum_{0}^{n-1}\left|a_{m}\right|^{2}+\left\|z^{n} T^{n} f\right\|_{\mathscr{H}}^{2}$ because $i_{\mathscr{H}}^{*} z^{m}=z^{m}, m=0, \ldots, n-1$. Therefore,

$$
\|g+f\|^{2}-\|f\|_{\mathscr{P}}^{2}=\left\|g+z^{n} T^{n} f\right\|^{2}-\left\|z^{n} T^{n} f\right\|_{\mathscr{P}}^{2},
$$

and the same expression holds for $\|g+\hat{f}\|^{2}-\|\hat{f}\|_{\mathscr{H}}^{2}$. Thus

$$
\|g\|_{\mathscr{M}}^{2}=\sup \left\{\|g+\hat{f}\|^{2}-\|\hat{f}\|_{\mathscr{H}}^{2}\right\}
$$

where the supremum is taken over all elements $\hat{f}$ of the kernel of $1-T^{n} T^{* n}$. Moreover, for these elements, $\|\hat{f}\|_{\mathscr{H}}=\left\|z^{n} \hat{f}\right\|_{\mathscr{H}}$ since $\left(1-T^{* n} T^{n}\right) z^{n} \hat{f}$ is simultaneously in the kernel of $T^{n}$ and orthogonal to the kernel of $T^{n}$. Indeed, for $m=0, \ldots, n-1,\left\langle\left(1-T^{* n} T^{n}\right) z^{n} \hat{f}, z^{m}\right\rangle_{\mathscr{H}}=\left\langle z^{n} \hat{f}, z^{m}\right\rangle_{\mathscr{H}}=\left\langle z^{n} \hat{f}, i_{\mathscr{H}}^{*} z^{m}\right\rangle_{\mathscr{H}}=$ $\left\langle z^{n} \hat{f}, z^{m}\right\rangle=0$. Consequently,

$$
\|g\|_{\mathscr{M}}^{2}=\sup \left\{\|g+\hat{f}\|^{2}-\left\|z^{n} \hat{f}\right\|_{\mathscr{H}}^{2}:\left(1-T^{n} T^{* n}\right) \hat{f}=0\right\} .
$$


Finally, since multiplication by $z$ is bounded by one on $\mathscr{M}$, and $\mathscr{H}=T^{n} \mathscr{H}$, it follows that

$$
\begin{aligned}
\left\|z^{n} g\right\|_{\mathscr{M}}^{2} & \leq\|z g\|_{\mathscr{M}}^{2} \\
& \leq\|g\|_{\mathscr{M}}^{2} \\
& =\sup \left\{\left\|g+T^{n} f\right\|^{2}-\left\|z^{n} T^{n} f\right\|_{\mathscr{P}}^{2}:\left(1-T^{n} T^{* n}\right) T^{n} f=0\right\} \\
& =\sup \left\{\left\|z^{n} g+f\right\|^{2}-\|f\|_{\mathscr{H}}^{2}:\left(1-T^{n} T^{* n}\right) T^{n} f=0\right\} \\
& \leq\left\|z^{n} g\right\|_{\mathscr{M}}^{2} .
\end{aligned}
$$

Therefore, multiplication by $z$ is isometric on $\mathscr{M}$.

Remark. The referee has kindly pointed out that other equivalent conditions for $\mathscr{H}=\mathscr{H}(b)$ isometrically were recently obtained by R. B. Leech [5].

\section{REFERENCES}

1. J. A. Ball and T. L. Kriete, III, Operator-valued Nevanlinna-Pick kernels and the functional models for contraction operators, Integral Equations Operator Theory 10 (1987), 17-61.

2. A. Beurling, On two problems concerning linear transformations in Hilbert space, Acta Math. 81 (1949), 239-255.

3. L. de Branges, Square summable power series (to appear).

4. L. de Branges and J. Rovnyak, Square summable power series, Holt, Rinehart and Winston, New York, 1966.

5. R. B. Leech, A characterization of a class of Hilbert spaces of power series, preprint.

6. N. K. Nikol'skii and V. I. Vasyunin, Notes on two function models, Proceedings of the Symposium on the Occasion of the Proof of the Bieberbach Conjecture, Math. Surveys, Amer. Math. Soc., Providence, 1986, pp. 113-141.

7. D. Sarason, Doubly shift-invariant spaces in $H^{2}$, J. Operator Theory 16 (1986), 75-97.

8. __ Shift-invariant spaces from the Brangesian point of view, Proceedings of the Symposium on the Occasion of the Proof of the Bieberbach Conjecture, Math. Surveys, Amer. Math. Soc., Providence, 1986, pp. 153-166.

Department of Mathematics, State University College of New York at Buffalo, Buffalo, New York 14222 